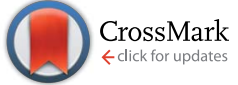

Cite this: RSC Adv., 2017, 7, 195

\title{
Sorption and photodegradation under visible light irradiation of an organic pollutant by a heterogeneous UiO-67-Ru-Ti MOF obtained by post-synthetic exchange $\dagger$
}

\author{
Ricardo Navarro Amador, Michaël Carboni* and Daniel Meyer
}

Received 9th November 2016 Accepted 23rd November 2016

DOI: $10.1039 / c 6 r a 26552 a$

www.rsc.org/advances

\begin{abstract}
The synthesis of a UiO-67 based MOF was made through the combination of two synthetic pathways, first a solvothermal synthesis in the presence of two different linkers, one for the structure and one to work as a light antenna (based on $\mathrm{Ru}$ ), followed by a process of post-synthetic metal exchange on the coordination node to include a catalyst (Ti). This MOF has been able to remove an organic pollutant from an aqueous solution and to catalyze the degradation of the pollutant under visible light irradiation.
\end{abstract}

\section{Introduction}

The efficient use of solar energy is one of the biggest challenges facing scientists; numerous research groups have dedicated their work to offer solutions for this technological purpose to have more ecological and environmentally friendly energy sources and industrial processes. For that matter, several studies have focused their attention on light-driven photochemical reactions that can use solar energy to perform the photo-degradation of industrial organic pollutants with light sensitive catalysts. These compounds are widely used in the textile industry and according to several studies ${ }^{1-3}$ textile dyes represent one of the largest groups of toxic organic compounds that contribute to water pollution. While some studies have shown promising results on the degradation and the sorption of these compounds, ${ }^{4-7}$ there is still the need to work specially on the energy conversion efficiency and on the harvesting capacities of the materials since the quantum yield is low due to the limitations of the usual materials for photo-catalysis. ${ }^{8}$ Moreover, a good number of the reported systems only work on the UV spectra of light which limits the application of these materials due to a low conversion yield. ${ }^{8}$

Metal-Organic Frameworks (MOFs) are a new class of porous coordination materials that have been studied for diverse applications, since the coining of the term by Yaghi and coworkers $^{9}$ in 1999. MOFs have been used in many applications as gas storage ${ }^{10}$ heterogeneous catalysis,${ }^{11}$ extraction, ${ }^{12}$ metals separation, ${ }^{13,14}$ drug delivery ${ }^{15}$ or as photocatalysts..$^{16}$ In the last example, MOFs have proven to have a great potential, mostly

ICSM, Institut de Chimie Séparative de Marcoule UMR 5257, CEA, CNRS, ENSCM, UM, Bât 426, BP 17171, 30207 Bagnols-sur-Cèze Cedex, France. E-mail: michael. carboni@cea.fr

$\dagger$ Electronic supplementary information (ESI) available. See DOI: $10.1039 / \mathrm{c} 6 \mathrm{ra} 26552 \mathrm{a}$ because during the synthesis of these materials the structure can be controlled to add a desired functionality in either the metallic cluster ${ }^{17}$ or in the organic ligand. ${ }^{18}$ This particularity allows scientist to synthesize materials with a photosensitizer and a catalytic center, all in the same crystalline structure which can be used as synthesized or can be also used as precursors to obtain porous metal oxides which can work as catalysts. ${ }^{19}$ Among the different photocatalytic MOFs that have been reported, zirconium and titanium based MOFs have attracted a lot of attention due to their high stability (thermal, mechanical or under irradiation) and their light excitation properties, respectively.

The family of the Zr-based MOFs, UiO, first reported by Lillerud and coworkers, ${ }^{20}$ has been one of the most studied MOFs in the last years due to its high stability. ${ }^{21}$ The size of the pore can vary along with the length of the iso-structural organic linkers. ${ }^{22,23}$ These properties led Lin and coworkers ${ }^{24}$ to incorporate different metal complexes of Ir, Re and Ru inside the structure of the UiO-67 by mixing during the synthesis two different ligands of similar length, the biphenyldicarboxylic acid and the metal complex anchored with the iso-structural 2,2'-bipyridine-5,5'-dicarboxylic acid. More recently, a similar material was studied by Morris and coworkers ${ }^{25-27}$ where they measured the photophysical properties of this MOF under visible light. The synthesis of heterometallic MOFs has also been achieved by the "Bifunctional Method" to obtain mixed materials based on the affinities of the reactants. ${ }^{28}$

Other interesting materials for photocatalysis are the $\mathrm{Ti}$ based MOFs, due to the photocatalytic properties and the low toxicity of Ti. The first Ti based MOF was the MIL-125 (ref. 29) that showed sensitivity to light under UV radiation. After, Li and coworkers ${ }^{18}$ developed an analogous of the MIL-125 where they functionalized the ligand with an amine group which allowed to shift the light absorption to the visible light spectra. However, 


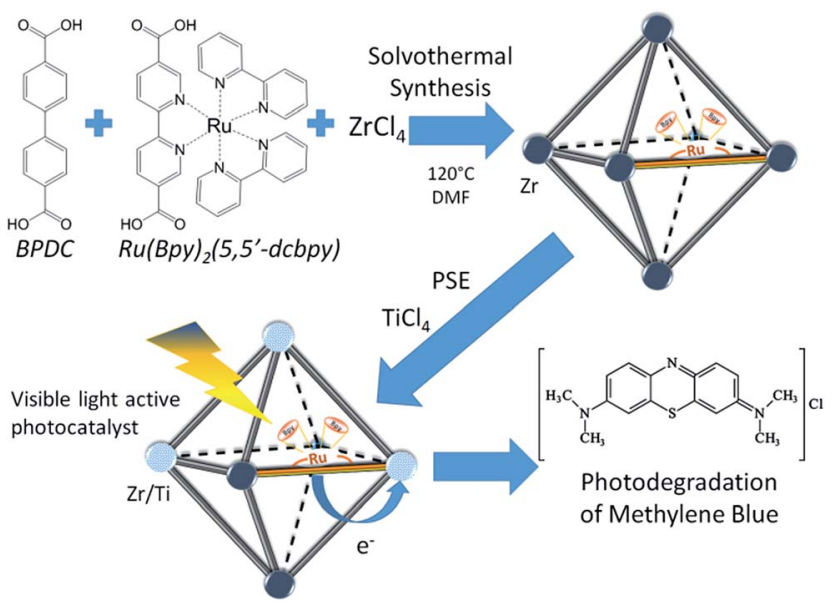

Fig. 1 Synthesis of photoactive UiO-67-Ru-TiXX MOF.

the synthesis of Ti based MOFs is still challenging and up to this date only few relevant Ti based MOFs have been reported as the NTU-9 (ref. 30) PCN-22, ${ }^{31}$ COK-69 (ref. 32) or the Ti-MIL-101. ${ }^{33}$

Another interesting approach to obtain heterometallic MOFs is the post-synthetic exchange (PSE) process. This technique allows to exchange partially either the metallic cluster or the organic ligand of a MOF without degradation of the MOF or the loss of crystallinity ${ }^{34}$ showing the flexibility and the microreversibility of the MOF structure. Recently, the process of PSE has been applied on the UiO-66 $\left(\mathrm{Zr}_{6} \mathrm{O}_{4}(\mathrm{OH})_{4} \mathrm{~L}_{6}, \mathrm{~L}=\right.$ terephtalic acid) to obtain a mixed Ti/Zr MOF. ${ }^{17,35,36}$

In this work, we propose the synthesis of a $\mathrm{Zr}$ UiO-67 based MOF doped with a Ru complex to work as a light antenna and with Ti included by PSE to be used as photocatalyst (Fig. 1). This methodology has allowed obtaining a multi-functional material that can work slightly under UV but more importantly that make $\mathrm{Ti}$ visible-light active through a $\mathrm{Ru}$ light antenna. Sorption capacity and photodegradation activity of the material were tested towards the degradation of methylene blue (MB) in aqueous solution, the results were compared with the capacities of the precursors MOFs: UiO-67, UiO-67-Ru (modified with $\mathrm{Ru}$ ) and UiO-67-Ti50 (modified with Ti). Synthesis of the modified UiO-67-UiO-67-Ru-TiXX ( $\mathrm{XX}=\%$ of Ti to $\mathrm{Zr}$ ) MOF was made through classical solvothermal synthesis coupled to a postsynthetic exchange (PSME) process.

\section{Experimental section}

Reagents were purchased from Sigma-Aldrich and were used as received. All ligands were fully characterized by ${ }^{1} \mathrm{H}$ NMR and were recorded at room temperature using a $400 \mathrm{MHz}$ spectrometer (Bruker). UV-visible analyses were recorded on a Shimadzu UV-36000 UV-Vis-NIR spectrometer with a diffuse reflectance measurement system. Spectrofluorometric properties were recorded and measured on a Horiba FluoroMax-4 Fluorimeter. PXRD patterns were obtained with a Bruker D8 Advance diffractometer. TGA was measured in a Mettler-Toledo TG with auto-sampler. Nitrogen physisorption measurements were performed using an ASAP 2020 at $77 \mathrm{~K}$, after outgassing at $363 \mathrm{~K}$ during 12 hours, reaching a pressure below $1 \mathrm{~mm} \mathrm{Hg}$, and specific surface areas were calculated using the BET method. ${ }^{37} \mathrm{~A}$ FEI Quanta 200 environmental scanning electron microscope, equipped with an Everhart-Thornley Detector (ETD) and a backscattered electron detector (BSED), was used to record images with an acceleration voltage of $30 \mathrm{kV}$ under high vacuum conditions. The chemical compositions of the solutions were measured by ICP-AES (Perkin Elmer Optima 2000 DV).

Synthesis of the modified UiO-67-UiO-67-Ru-TiXX ( $\mathrm{XX}=$ mol\% of Ti/Zr) MOF was made through classical solvothermal synthesis coupled to a post-synthetic exchange process (Fig. 1). Details on the synthesis conditions and the characterization of complexes and ligands are provided within the ESI. $\dagger$

\subsection{Synthesis of UiO-67 and UiO-67-Ru}

A solution of $\mathrm{ZrCl}_{4}(0.065 \mathrm{mg}, 0.28 \mathrm{mmol})$ in dry DMF $(5 \mathrm{~mL})$ was prepared in a glass vial. To this solution it was added water (13 $\mu \mathrm{L})$ and the mixture was sonicated during 10 minutes. Then, it was added benzoic acid (1.05 g, $8.6 \mathrm{mmol}), 4,4^{\prime}$-biphenyldicarboxylic acid (bpdc) (65 mg, $0.27 \mathrm{mmol}$ ) and in the case of the Ru doped MOF Ru(bpy) $)_{2}\left(5,5^{\prime}\right.$-dcbpy) (13.4 mg, $\left.0.02 \mathrm{mmol}\right)$. The mixture was sonicated during 5 minutes before the addition of each of the reactants. The vials were then placed in an oven in static conditions at $120{ }^{\circ} \mathrm{C}$ during 72 hours. The obtained precipitate was separated by centrifugation and was washed 3 times with DMF, 3 times with ethanol and finally 2 times with diethyl ether. The crystalline solid was characterized by ICP, TGA, BET and PXRD.

\subsection{PSME with $\mathrm{TiCl}_{4} \mathrm{THF}$}

UiO-67 or UiO-67-Ru was placed in a glass vial by adding $10 \mathrm{mg}$ of MOF into $1 \mathrm{~mL}$ of a solution of $\mathrm{TiCl}_{4}$ THF in DMF $(9 \mathrm{mg}$ $\left.\mathrm{mL}^{-1}\right)$. The mixture was sonicated and placed in an oven at $120{ }^{\circ} \mathrm{C}$ for 6 days. Two times a day the mixture was shaken. The solid was isolated by centrifugation and washed 3 times with fresh DMF, with ethanol, with diethyl ether and finally dried in an oven at $40{ }^{\circ} \mathrm{C}$ overnight. The crystalline solid was characterized by ICP, TGA, BET and PXRD.

\subsection{Dye uptake measurements}

$5 \mathrm{mg}$ of dry MOF (UiO-67, UiO-67-Ti, UiO-67-Ru, UiO-67-Ru-Ti) were added to $5 \mathrm{~mL}$ of an aqueous saturated solution of $\mathrm{MB}$. The mixtures were stirred in the dark until equilibrium was reached. Aliquots of the solution were taken in between to follow the variation of the dye concentration. The solutions were centrifuged to isolate the solid MOF from the liquid and the concentration was followed by UV-Vis spectroscopy. MB calibration curve was obtained by measuring the samples with different concentrations (Fig. S3†).

\subsection{Dye photo-degradation}

$5 \mathrm{mg}$ of each MOF (UiO-67, UiO-67-Ti, UiO-67-Ru, UiO-67-RuTi) were added to $5 \mathrm{~mL}$ of an aqueous solution of $\mathrm{MB}$ with a known concentration. The mixtures were stirred in the dark 
overnight to achieve adsorption equilibrium of the dye in the MOF. Then, the samples were irradiated during 4 hours under UV $(250 \mathrm{~nm})$ or visible light $(419.5 \mathrm{~nm})$ in a Rayonet RPR-100 Photochemical Reactor (400 Watts approximately). The concentration of methylene blue was determined by UV-Visible spectroscopy.

\section{Results and discussion}

The efficiency of the exchange was qualified by XRF (Fig. S1 $\dagger$ ) and quantified by ICP-AES after digestion of the material (view ESI $\dagger$ for digestion procedure). Values for different MOFs are shown in Table 1. It has been possible to add up to a $50 \%$ of $\mathrm{Ti}$ in comparison to the amount of $\mathrm{Zr}$ depending on the concentration of the $\mathrm{TiCl}_{4}$ solution used for the PSE. The percentage of Ti in the MOF was graphed against the concentration of the original concentration and a linear tendency was observed (Fig. S3†). The selected concentration for further characterization was the material with an exchange of $50 \%$ of titanium (UiO-67-Ru-Ti50) due to the maximum value of exchange without loss of crystallinity that have been observed for the UiO- $66 .{ }^{38}$

The amount of $\mathrm{Ru}$ inside the material was also quantified by ICP-AES with the digested material. The incorporation of $\mathrm{Ru}$ complexes is low in comparison to the amount of exchange $\mathrm{Ti} /$ $\mathrm{Zr}$ (around $5 \%$ if compared to the ligand). This low

Table 1 Concentration of $\mathrm{Ti}$ and Ru in different MOFs

\begin{tabular}{llll}
\hline & $\begin{array}{l}\text { Concentration } \\
\text { of } \mathrm{TiCl}_{4} \text { in } \\
\text { exchange solution } \\
\left(\mathrm{mg} \mathrm{mL}^{-1}\right)\end{array}$ & $\begin{array}{l}\text { Ratio of } \\
\mathrm{Ti} / \mathrm{Zr} \text { in } \\
\text { MOF\% }\end{array}$ & $\begin{array}{l}\text { \% Ru with } \\
\text { respect to the } \\
\text { ligand }\end{array}$ \\
Samples & 9.0 & 54.5 & 4.0 \\
\hline UiO-67-Ru-Ti50 & 3.6 & 21.0 & 4.0 \\
UiO-67-Ru-Ti20 & 3.6 & 12.9 & 4.1 \\
UiO-67-Ru-Ti10 & 1.8 & &
\end{tabular}

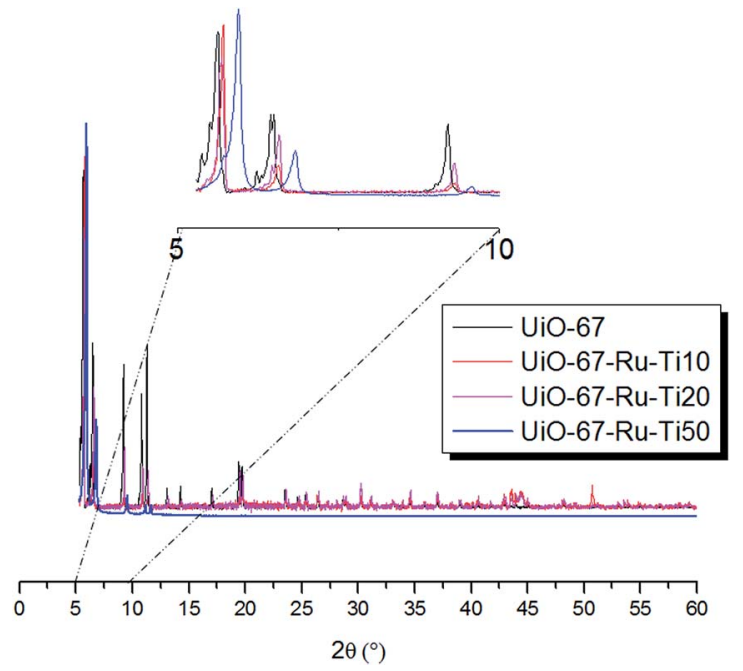

Fig. 2 PXRD patterns for UiO-67-Ru (black) and UiO-67-Ru-Ti with different loadings of Ti MOFs (red, purple and blue).

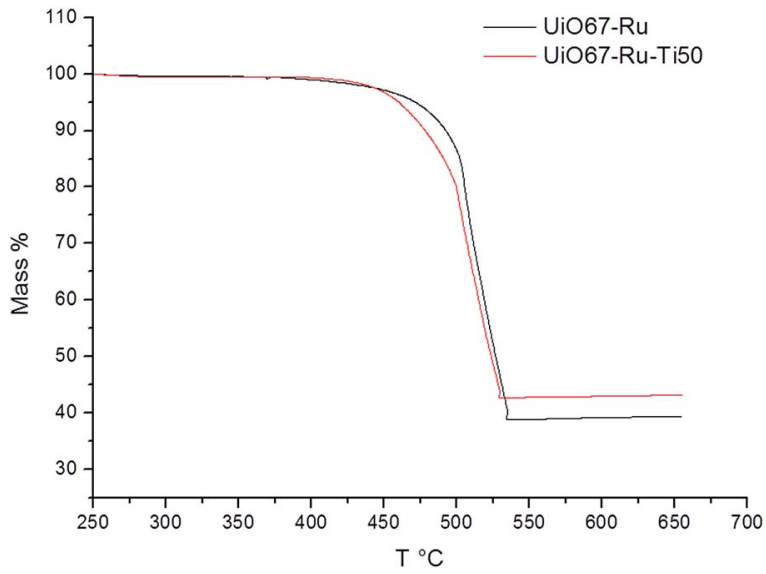

Fig. 3 TGA of $\mathrm{UiO}-67-\mathrm{Ru}$ and $\mathrm{UiO}-67-\mathrm{Ru}-\mathrm{Ti} 50$.

incorporation is to avoid effects on the crystalline structure of the MOF and to oversaturate the photo-activity of the MOF to ensure transference of the electrons to the catalytic centers.

PXRD analyses allowed to verify the crystallinity of the materials before and after the exchange with different concentrations of Ti. In Fig. 2 are shown the signals measured with the UiO-67 and UiO-67-Ru-Ti 10, 20 and 50. Even in low\% of Ti exchange a small shift is noticeable for low values of $2 \theta$ that can be explained by the contraction of the cavities due to the change of size from $\mathrm{Zr}$ to Ti. This shift is enhanced with higher loadings of Ti indicating that part of the $\mathrm{Zr}$ in the cluster is indeed substituted by Ti as previously reported in other studies. ${ }^{38}$

TGA results (Fig. 3) were analyzed to obtain the relation between the organic and the inorganic part for the UiO-67-Ru and UiO-67-Ru-Ti50 MOFs. The analysis of UiO-67-Ru-Ti50 shows that the percentage value of the inorganic residues increased after the exchange. This fact indicates that Ti is not only exchanging part of the cluster as shown by PXRD, but it can also be found inside the cavities of the MOF or coordinate to the cluster, since for a total exchange it would be expected a decrease of the inorganic residues (Table S1†). All the materials showed a good thermal stability with a decomposition temperature above $450{ }^{\circ} \mathrm{C}$ with no significant changes between the exchanged and the un-exchanged materials. BET surface was measured for MOFs UiO-67-Ru and UiO-67-Ru-Ti50 (Fig. S4 and S5 $\dagger$ ), the results show a decrease on the surface area after the metal exchange from $1847 \mathrm{~m}^{2} \mathrm{~g}^{-1}$ to $1694 \mathrm{~m}^{2} \mathrm{~g}^{-1}$. This is in agreement with the TGA analysis that part of the pores is occupied by the Ti. Finally, SEM images (Fig. S6 $\dagger$ ) corresponding to the different materials have the typical octahedral structure of the UiO-67; along with the SEM, it was performed the EDS analysis of the surface, however the ratio of $\mathrm{Zr}$ to $\mathrm{Ti}$ obtained showed a decrease if compared to the values obtained by ICP indicating that Ti is mainly inside the MOF structure than on the surface (Table S2 $\dagger$ ).

Photocatalytic properties of the materials were measured by UV-Visible analysis and Fluorimetry. UV-visible analysis (Fig. 4) showed an increase in the signals obtained between 250 and $300 \mathrm{~nm}$, values indicating a similar activity to Ti oxides in the MOF as reported in previous works. ${ }^{36}$ 


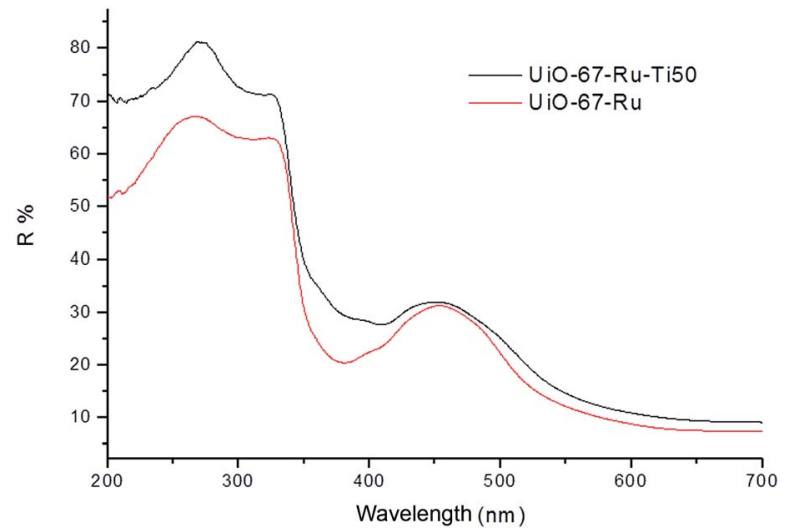

Fig. 4 UV-Vis spectra of UiO-67-Ru and UiO-67-Ru-Ti50.

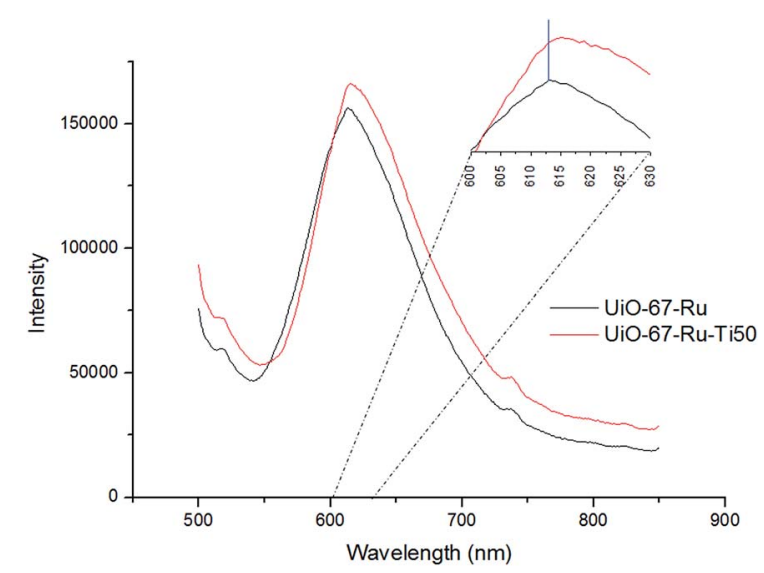

Fig. 5 Emission spectra of UiO-67-Ru and UiO-67-Ru-Ti50.

The Fluorimetry Analysis allowed to identify the characteristic peak of emission of Ru complexes inside the MOF. Previous characterization of the $\mathrm{Ru}(\mathrm{bpy})_{3}$ complexes showed that the emission maximum of these compounds is at $621 \mathrm{~nm}$, while the UiO-67-Ru MOF showed a small shift to lower values of $613 \mathrm{~nm}$ and the exchanged UiO-67-Ru-Ti50 MOF showed a maximum of absorption at $615 \mathrm{~nm}$ (Fig. 5).

Lifetime measurements were performed for the UiO-67-Ru MOF and the UiO-67-Ru-Ti50, showing a decrease on the value with the exchanged MOF. UiO-67-Ru showed a lifetime value of $3.43 \times 10^{-7}$ seconds while the exchanged material showed a value of $4.52 \times 10^{-8}$ seconds (Fig. S7†). The decrease on this value can be related to the proximity of $\mathrm{Ru}$ to the Ti catalytic center. Also, the fit was obtained with a mono-exponential fitting, indicating the presence of just one type of light interaction of the Ru complex in the MOF.

Dye sorption results are shown in Fig. S8. $\dagger$ With the introduction of $\mathrm{Ru}$ and $\mathrm{Ti}$ respectively, the sorption decreases considerably due to some obstructions of the MOF cavities by the Ru complex and the addition of Ti.

The adsorption data obtained was fitted to a pseudo-first and pseudo-second-order rate equations. The linear pseudo first order is represented by

$$
\ln \left(q_{\mathrm{e}}-q_{t}\right)=\ln \left(q_{\mathrm{e}}\right)-k_{1} t
$$

The linear form of the pseudo-second-order rate equation is expressed as

$$
\frac{t}{q_{t}}=\frac{1}{k_{2} q_{\mathrm{e}}{ }^{2}}+\frac{t}{q_{\mathrm{e}}}
$$

where $q_{\mathrm{e}}$ is the sorption capacity at equilibrium, $q_{t}$ is the sorption capacity at a given time; $k_{1}$ and $k_{2}$ are the pseudo-first and pseudo-second-order rate constants, respectively. Fittings are presented in Fig. S9 and S10. $\dagger$ A better fitting is observed with the second order equation. In Tables 2 and $\mathrm{S} 3 \uparrow$ are summarized the kinetic parameters for the pseudo-first and pseudo second order kinetics parameters.

The diffusion speed also seems to be affected, by prolonging the time to reach equilibrium in any of the modified MOFs, either with $\mathrm{Ti}$ or $\mathrm{Ru}$ or the combination of both.

The photo-degradation results are shown in Fig. S11 $\uparrow$ and 6 under UV and visible irradiation respectively for the different synthesized materials. It can be observed a decrease on the concentration of dyes especially with UiO-67-Ru-Ti50. This shows the increase on the photo-activity as well as on the kinetics of the degradation reaction in comparison to its precursors. These results indicate that the photocatalytic reaction is boosted by the communication of the Ru with the Ti, while the reaction takes place on the Ti centers in the MOF. This can be concluded since the activity is almost inexistent in any of the other materials (UiO-67, UiO-67-Ru or UiO-67-Ti). The catalytic degradation of MB with Ti oxides has been described in several studies ${ }^{39}$ and it is reported that this reaction takes places through the generation of $\cdot \mathrm{OH}$ radicals that can react directly with adsorbed dye molecules. An equivalent mechanism has been proposed for the degradation of $\mathrm{MB}$ with different MOFs ${ }^{4,40,41}$ where electrons are transferred from the valence to the conduction band that allow the generation of the $\cdot \mathrm{OH}$ that has the ability of reacting with azo dyes to decompose them. A scheme of the possible products from the degradation of $\mathrm{MB}$ is provided within the ESI (Fig. S12†).

\begin{tabular}{|c|c|c|c|c|}
\hline Parameter & UiO-67 & $\mathrm{UiO}-67-\mathrm{Ru}$ & UiO-67-Ti50 & UiO-67-Ru-Ti50 \\
\hline$q_{\mathrm{e}}(\exp ).\left(\mathrm{mg} \mathrm{g}^{-1}\right)$ & 257 & 104 & 70.5 & 40.4 \\
\hline$k_{2}\left[\mathrm{~g}\left(\mathrm{mg}^{-1} \min ^{-1}\right)\right]$ & $\begin{array}{l}2.28 \times 10^{-5} \pm 9.45 \\
\times 10^{-7}\end{array}$ & $\begin{array}{l}5.03 \times 10^{-5} \pm 9.89 \times \\
10^{-6}\end{array}$ & $\begin{array}{l}2.62 \times 10^{-4} \pm 7.53 \times \\
10^{-5}\end{array}$ & $\begin{array}{l}1.06 \times 10^{-3} \pm 2.43 \times \\
10^{-4}\end{array}$ \\
\hline$q_{\mathrm{e}}($ calc. $)\left(\mathrm{mg} \mathrm{g}^{-1}\right)$ & $285 \pm 3.83$ & $111 \pm 3.77$ & $72.9 \pm 2.58$ & $42.0 \pm 1.52$ \\
\hline$R^{2}$ & 0.998 & 0.992 & 0.993 & 0.993 \\
\hline
\end{tabular}

Table 2 Pseudo-second order sorption kinetic parameters for methylene blue on different MOFs 


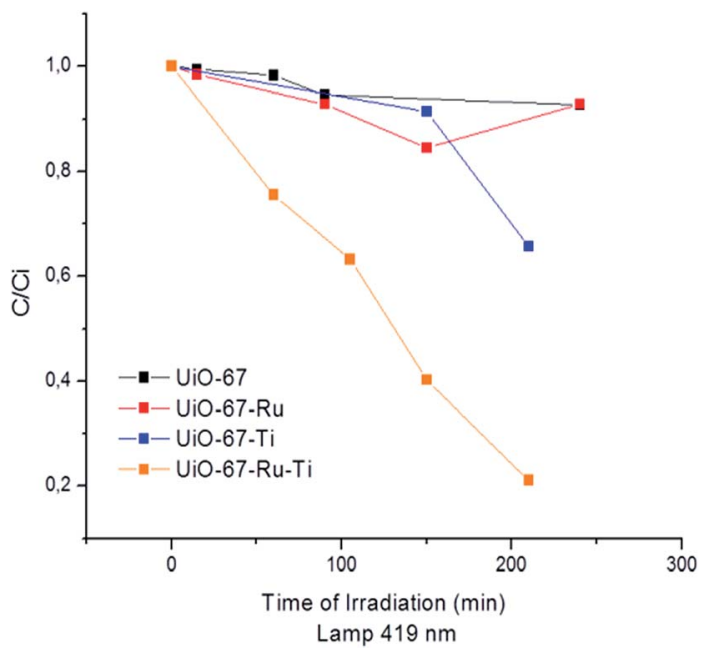

Fig. 6 Photo-degradation of MB under visible irradiation $(419 \mathrm{~nm})$.

\section{Conclusions}

A mixed MOF with photocatalytic activity under visible light has been developed by an original pathway. The material preserves the general structural characteristics of the Zr UiO family while adding Ru complexes and the catalytic advantages of Ti. This material has shown to be active towards the degradation of methylene blue with a good improvement due to the modifications on the structure even when the exchange is not complete, proving the interaction between the light antenna and the catalytic center, since the materials with just one of the mentioned modifications did not show a big improvement on the catalytic activity. Finally, this strategy shows that new materials can be obtained by combining different methodologies of synthesis to add multiple constituents in the same structure, each one with a specific role to enhance the overall properties of the material.

\section{Acknowledgements}

The authors are grateful to Joseph Lantru for the SEM analyses and Bruno Corso for the PXRD measurements. RNA thanks CONACYT for the scholarship 382043.

\section{Notes and references}

1 I. K. Konstantinou and T. A. Albanis, Appl. Catal., B, 2004, 49, 1-14.

2 A. Ajmal, I. Majeed, R. N. Malik, H. Idriss and M. A. Nadeem, RSC Adv., 2014, 4, 37003-37026.

3 E. Haque, J. W. Jun and S. H. Jhung, J. Hazard. Mater., 2011, 185, 507-511.

4 J.-J. Du, Y.-P. Yuan, J.-X. Sun, F.-M. Peng, X. Jiang, L.-G. Qiu, A.-J. Xie, Y.-H. Shen and J.-F. Zhu, J. Hazard. Mater., 2011, 190, 945-951.

5 K. G. M. Laurier, F. Vermoortele, R. Ameloot, D. E. De Vos, J. Hofkens and M. B. J. Roeffaers, J. Am. Chem. Soc., 2013, 135, 14488-14491.
6 S. Pu, L. Xu, L. Sun and H. Du, Inorg. Chem. Commun., 2015, $52,50-52$.

7 P. Yu, Q. Li, Y. Hu, N. Liu, L. Zhang, K. Su, J. Qian, S. Huang and M. Hong, Chem. Commun., 2016, 52, 7978-7981.

8 F. Fresno, R. Portela, S. Suárez and J. M. Coronado, J. Mater. Chem. A, 2014, 2, 2863-2884.

9 H. Li, M. Eddaoudi, M. O'Keeffe and O. M. Yaghi, Nature, 1999, 402, 276-279.

10 D. J. Tranchemontagne, K. S. Park, H. Furukawa, J. Eckert, C. B. Knobler and O. M. Yaghi, J. Phys. Chem. C, 2012, 116, 13143-13151.

11 M. I. Gonzalez, E. D. Bloch, J. A. Mason, S. J. Teat and J. R. Long, Inorg. Chem., 2015, 54, 2995-3005.

12 M. Carboni, C. W. Abney, S. Liu and W. Lin, Chem. Sci., 2013, 4, 2396-2402.

13 E. Perez, R. Navarro Amador, M. Carboni and D. Meyer, Mater. Lett., 2016, 167, 188-191.

14 E. Perez, M.-L. Andre, R. Navarro Amador, F. Hyvrard, J. Borrini, M. Carboni and D. Meyer, J. Hazard. Mater., 2016, 317, 617-621.

15 K. M. L. Taylor-Pashow, J. D. Rocca, Z. Xie, S. Tran and W. Lin, J. Am. Chem. Soc., 2009, 131, 14261-14263.

16 R. Navarro Amador, M. Carboni and D. Meyer, Mater. Lett., 2016, 166, 327-338.

17 H. G. T. Nguyen, L. Mao, A. W. Peters, C. O. Audu, Z. J. Brown, O. K. Farha, J. T. Hupp and S. Nguyen, Catal. Sci. Technol., 2015, 5, 4444-4451.

18 Y. Fu, D. Sun, Y. Chen, R. Huang, Z. Ding, X. Fu and Z. Li, Angew. Chem., Int. Ed., 2012, 51, 3364-3367.

19 T.-T. Li, J. Qian and Y.-Q. Zheng, $R S C A d v .$, 2016, 6, 7735877365.

20 J. H. Cavka, S. Jakobsen, U. Olsbye, N. Guillou, C. Lamberti, S. Bordiga and K. P. Lillerud, J. Am. Chem. Soc., 2008, 130, 13850-13851.

21 C. Wang, X. Liu, N. Keser Demir, J. P. Chen and K. Li, Chem. Soc. Rev., 2016, 5107-5134.

22 M. Kim and S. M. Cohen, CrystEngComm, 2012, 14, 40964104.

23 M. Kandiah, M. H. Nilsen, S. Usseglio, S. Jakobsen, U. Olsbye, M. Tilset, C. Larabi, E. A. Quadrelli, F. Bonino and K. P. Lillerud, Chem. Mater., 2010, 22, 6632-6640.

24 C. Wang, Z. Xie, K. E. deKrafft and W. Lin, J. Am. Chem. Soc., 2011, 133, 13445-13454.

25 W. A. Maza, S. R. Ahrenholtz, C. C. Epley, C. S. Day and A. J. Morris, J. Phys. Chem. C, 2014, 118, 14200-14210.

26 W. A. Maza and A. J. Morris, J. Phys. Chem. C, 2014, 118, 8803-8817.

27 W. A. Maza, R. Padilla and A. J. Morris, J. Am. Chem. Soc., 2015, 137, 8161-8168.

28 J. Qian, Q. Li, L. Liang, Y. Yang, Z. Cao, P. Yu, S. Huang and M. Hong, Chem. Commun., 2016, 52, 9032-9035.

29 M. Dan-Hardi, C. Serre, T. Frot, L. Rozes, G. Maurin, C. Sanchez and G. Férey, J. Am. Chem. Soc., 2009, 131, 10857-10859.

30 J. Gao, J. Miao, P.-Z. Li, W. Y. Teng, L. Yang, Y. Zhao, B. Liu and Q. Zhang, Chem. Commun., 2014, 50, 3786. 
31 S. Yuan, T.-F. Liu, D. Feng, J. Tian, K. Wang, J. Qin, Q. Zhang, Y.-P. Chen, M. Bosch, L. Zou, S. Teat, S. Dalgarno and H.-C. Zhou, Chem. Sci., 2015, 6, 3926-3930.

32 B. Bueken, F. Vermoortele, D. E. P. Vanpoucke, H. Reinsch, C.-C. Tsou, P. Valvekens, T. De Baerdemaeker, R. Ameloot, C. E. A. Kirschhock, V. Van Speybroeck, J. M. Mayer and D. De Vos, Angew. Chem., Int. Ed., 2015, 54, 13912-13917.

33 J. A. Mason, L. E. Darago, W. W. Lukens and J. R. Long, Inorg. Chem., 2015, 54, 10096-10104.

34 S. M. Cohen, Chem. Rev., 2012, 112, 970-1000.

35 S. J. D. Smith, B. P. Ladewig, A. J. Hill, C. H. Lau and M. R. Hill, Sci. Rep., 2015, 5, 7823-7828.
36 Y. Lee, S. Kim, J. K. Kang and S. M. Cohen, Chem. Commun., 2015, 51, 5735-5738.

37 S. Brunauer, P. H. Emmett and E. Teller, J. Am. Chem. Soc., 1938, 60, 309-319.

38 D. Sun, W. Liu, M. Qiu, Y. Zhang and Z. Li, Chem. Commun., 2015, 51, 2056-2059.

39 U. G. Akpan and B. H. Hameed, J. Hazard. Mater., 2009, 170, 520-529.

40 H.-P. Jing, C.-C. Wang, Y.-W. Zhang, P. Wang and R. Li, RSC $A d v .$, 2014, 4, 54454-54462.

41 C.-F. Zhang, L.-G. Qiu, F. Ke, Y.-J. Zhu, Y.-P. Yuan, G.-S. Xu and X. Jiang, J. Mater. Chem. A, 2013, 1, 14329. 